constructions in [3]. I am grateful to I. Kaplansky and O. F. G. Schilling for discussion and correspondence concerning Theorem 2.

\title{
REFERENCES
}

1. E. Artin, Algebraic numbers and algebraic functions, I, Mimeographed lecture notes, Princeton University, New York University, 1951.

2. I. Kaplansky, Maximal fields with valuations, Duke Math. J. vol. 9 (1942) pp. 303-321.

3. O. F. G. Schilling, Arithmetic in fields of formal power series in several variables, Ann. of Math. vol. 38 (1937) pp. 551-576.

4. - The theory of valuations, Math. Surveys, no. 4, American Mathematical Society, New York, 1950.

5. G. Whaples, Generalized local class field theory, I and II, Duke Math. J. vol. 19 (1952) pp. 505-517, and vol. 21 (1954) pp. 247-256.

6. $\longrightarrow$, Existence of generalized local class fields, Proc. Nat. Acad. Sci. U.S.A. vol. 39 (1953) pp. 1100-1103.

INDIANA UNIVERSITY AND

INSTITUTE FOR ADVANCED StUdY

\section{A NOTE ON COMPLETELY PRIMARY RINGS}

\section{S. J. BRYANT AND J. L. ZEMMER}

A completely primary ring will mean a commutative ring with identity in which the ideal of nilpotent elements, called the radical, is a maximal ideal. For a completely primary ring $A$ with radical $N$, $\bar{A}$ will mean the quotient ring $A / N$. It has been shown by E. Snapper ${ }^{1}$ that if $A$ is a completely primary ring of characteristic zero then $A$ contains a field $F$ isomorphic with $\bar{A}$. The purpose of this note is to extend and, incidentally, give another proof of Snapper's result.

THEOREM. If $A$ is a completely primary ring of characteristic zero and $N$ its radical, then $A$ contains a field $F$ isomorphic with $\bar{A}=A / N$ such that each $a$ in $A$ can be uniquely written in the form $a=f+n$, where $f \in F, n \in N$.

Proof. First note that $x \notin N$ implies that $x$ has an inverse, $x^{-1}$. By Zorn's lemma $A$ contains a maximal ring $F$ whose intersection with $N$ is 0 . This ring $F$ is a field, for otherwise the set $F^{*}$ of all elements of the form $a b^{-1}, 0 \neq b, a \in F$, is a field containing $F$, whose intersection with $N$ is 0 , a contradiction. To prove the theorem it is sufficient to show that $A$ is identical with the subset $A^{*}$ of $A$ consisting of all

Received by the editors April 10, 1956.

${ }^{1}$ E. Snapper, Completely primary rings, III Imbedding and isomorphism theorems, Ann. of Math. vol. 53 (1951) pp. 207-234. See Remark 4.1, p. 218. 
elements of the form $f+n, f \in F, n \in N$. Assume the contrary, then there is an $x \in A$ such that $x \in A^{*}$. For $z \in A$ denote by $\bar{z}$ the image in $\bar{A}$ of $z$ under the natural homomorphism of $A$ onto $\bar{A}$, and by $\bar{F}$ the set of all $\bar{f}, f \in F$. Note that $\bar{F}$ is a subfield of $\bar{A}$ isomorphic with $F$. Since $x \notin A^{*}, \bar{x} \neq \overline{0}$ and $\bar{x} \in \bar{F}$. Suppose that $\bar{x}$ is transcendental over $\bar{F}$. Then for any element $g=f_{0}+f_{1} x+\cdots+f_{n} x^{n}$, in the subring $F[x]$ of $A$ generated by $F$ and $x$, it is clear that $g \in N$ implies $g=0$, or $N \cap F[x]=0$. This, however, contradicts the maximality of $F$, since $F[x] \supset F$. Thus, $\bar{x}$ must be algebraic over $\bar{F}$.

Now, let $\bar{f}(\lambda)=\bar{f}_{0}+\bar{f}_{1} \lambda+\cdots+\bar{f}_{m} \lambda^{m}$ be a minimum polynomial for $\bar{x}$ over $\bar{F}$. Denote by $n(r)$ the element $f_{0}+f_{1}(x+r)+\cdots+f_{m}(x+r)^{m}$ in the subring $F_{r}$ of $A$ generated by $F$ and $x+r$, and note that for each $r \in N, n(r) \in N$. Further, if $h=h_{0}+h_{1}(x+r)+\cdots+h_{t}(x+r)^{t}$ $\in F_{r}$, with $t<m$, then $h \in N$. It will be shown that for some $r \in N$, $n(r)=0$. Assume the contrary and choose $r_{0}$ so that $n\left(r_{0}\right)$ has minimum exponent $\rho>1$. Let $x_{1}=x+r_{0}$ and $f^{(i)}\left(x_{1}\right)=a_{0}+a_{1} x_{1}+\cdots$ $+a_{m-i} x_{1}^{m-i}$, where $a_{j}=(i+j)(i+j-1) \cdots(j+1) f_{i+j} \in F$. In particular, $f^{\prime}\left(x_{1}\right)=f_{1}+2 f_{2} x_{1}+\cdots+m f_{m} x_{1}^{m-1} \notin N$, and hence has an inverse in $A$. Let $r_{1}=-\left[f^{\prime}\left(x_{1}\right)\right]^{-1} n\left(r_{0}\right)$, so that

$$
\begin{aligned}
n\left(r_{0}+r_{1}\right) & =f_{0}+f_{1}\left(x+r_{0}+r_{1}\right)+\cdots+f_{m}\left(x+r_{0}+r_{1}\right)^{m} \\
& =n\left(r_{0}\right)+f^{\prime}\left(x_{1}\right) r_{1}+\frac{1}{2 !} f^{(2)}\left(x_{1}\right) r_{1}^{2}+\cdots+\frac{1}{m !} f^{(m)}\left(x_{1}\right) r_{1}^{m} \\
& =\left[\frac{1}{2 !} f^{(2)}\left(x_{1}\right)+\cdots+\frac{1}{m !} f^{(m)}\left(x_{1}\right) r_{1}^{m-2}\right] r_{1}^{2}=c\left[n\left(r_{0}\right)\right]^{2},
\end{aligned}
$$

has exponent less than $\rho$. This is a contradiction and hence $n\left(r_{0}\right)=0$.

Consider next the subring $F_{r_{0}}$ of $A$. Recall that this is the subring generated by $F$ and $x_{1}=x+r_{0}$. Since $f\left(x_{1}\right)=n\left(r_{0}\right)=0$ it is clear that the kernel of the homomorphism, $g(\lambda) \rightarrow g\left(x_{1}\right)$, of the polynomial domain $F[\lambda]$ onto $F_{r_{0}}$ is the prime ideal $(f(\lambda))$. Thus $F_{r_{0}}$ is a field and hence $N \cap F_{r_{0}}=0$. Finally, $x \notin A *$ implies $x_{1}=x+r_{0} \notin A$, so that $F_{r_{0}}$ properly contains $F$, contrary to the maximality of $F$. This completes the proof of the theorem.

UNIVERSITY OF MISSOURI 